\title{
Disseminated Exophiala dermatitidis causing septic arthritis and osteomyelitis
}

\author{
Raynell Lang ${ }^{1}$, Jessica Minion², Stuart Skinner ${ }^{3,4}$ and Alexander Wong ${ }^{3,4^{*}}$
}

\begin{abstract}
Background: Exophiala dermatitidis is a melanized fungus isolated from many environmental sources. Infections caused by Exophiala species are typically seen in immunocompromised hosts and manifest most commonly as cutaneous or subcutaneous disease. Systemic infections are exceedingly rare and associated with significant morbidity and mortality

Case presentation: A 28-year-old female originally from India presented with fevers, chills, weight loss and increasing back pain. She had a recent diffuse maculopapular rash that resulted in skin biopsy and a tentative diagnosis of sarcoidosis, leading to administration of azathioprine and prednisone. An MRI of her spine revealed a large paraspinal abscess requiring surgical intervention and hardware placement. Cultures from the paraspinal abscess grew a colony of dark pigmented mold. Microscopy of the culture revealed a melanized fungus, identified as Exophiala dermatitidis. Voriconazole was initially utilized, but due to relapse of infection involving the right iliac crest and left proximal humerus, she received a prolonged course of amphotericin B and posaconazole in combination and required 7 separate surgical interventions. Prolonged disease stability following discontinuation of therapy was achieved.

Conclusions: Described is the first identified case of disseminated Exophiala dermatitidis causing osteomyelitis and septic arthritis in a patient on immunosuppressive therapy. A positive outcome was achieved through aggressive surgical intervention and prolonged treatment with broad-spectrum antifungal agents.
\end{abstract}

Keywords: Exophiala dermatitidis, Phaeohyphomycosis, Wangiella dermatitidis, Disseminated, Septic arthritis, Osteomyelitis

\section{Background}

Exophiala dermatitidis is a melanized fungus isolated from many environmental sources [1]. It is a saprobe, using extracellular digestion to obtain nutrients from dead or decaying organic matter [2, 3]. Infections caused by Exophiala species are known as phaeohyphomycosis, due to the appearance of dark pigmented, irregular branching, septate hyphae [1-5]. Infections are typically seen in immunocompromised hosts such as transplant recipients and manifest as subcutaneous disease [6,7]. Systemic infections are rare and associated with significant morbidity and mortality $[5,8,9]$.

We describe, to our knowledge, the first case of disseminated $E$. dermatitidis with widespread involvement

\footnotetext{
* Correspondence: alexander.wong@usask.ca

${ }^{3}$ Division of Infectious Diseases, Department of Medicine, University of

Saskatchewan, Regina, Saskatchewan, Canada

${ }^{4} 4 \mathrm{E}$ - ID Clinic, Regina General Hospital, 1440 14th Avenue Regina, Regina,

Saskatchewan, Canada

Full list of author information is available at the end of the article
}

including osteomyelitis and septic arthritis in a previously healthy 28-year-old female on immunosuppressive therapy. A positive outcome was achieved through aggressive surgical intervention and prolonged treatment with broad-spectrum antifungal agents.

\section{Case presentation}

A previously healthy 28-year-old female presented with complaints of increasing back pain and fevers. Originally from India, she had immigrated to Canada two years prior and within months of arrival, developed fevers associated with unintentional weight loss, and a diffuse rash localized to her torso, legs and scalp. The rash was annular, with dark-colored plaques and central clearing. Skin biopsy identified granulomatous inflammatory cell infiltrates with staining negative for mycobacteria and fungus. A diagnosis of sarcoidosis was suggested. The lesions resolved spontaneously within six months with no specific therapy, but her fevers and weight loss continued. 
The patient elected to return to India for further investigations and treatment of her ongoing symptoms. Diffuse lymphadenopathy was identified and an inguinal lymph node biopsy revealed non-caseating granulomatous changes. Mycobacterial and fungal stains were negative. Brucella IgM and IgG serology were positive, but serum agglutination testing (SAT) to detect antibodies against the smooth lipopolysaccharide (S-LPS) of the outer membrane was negative. While in India, she received empiric treatment for tuberculosis and brucellosis with isoniazid, rifampin, pyrazinamide, ethambutol and injectable streptomycin. After nearly eight weeks of therapy, she experienced no clinical change. Her antimicrobials were discontinued, and she was started on high dose prednisone with rapid resolution of her malaise and fevers. She returned to Canada with a presumptive diagnosis of sarcoidosis on hydroxychloroquine, azathioprine, and tapering prednisone initiating at $40 \mathrm{mg}$ daily.

Shortly after her return, the patient developed a large nodule on her back with progressive back pain, worsening left arm pain, and ongoing fevers. She was admitted to hospital for further testing. Hepatosplenomegaly was noted with no appreciable lymphadenopathy. A tender mass was identified medial to her left scapula. She had no focal neurologic deficits and the remainder of her physical exam was non-contributory.

Investigations revealed a white blood cell (WBC) count of $14.3 \times 10^{9}$ per liter (L) and a platelet count of $885 \times 10^{9} / \mathrm{L}$. Her liver enzymes and creatinine kinase were within normal limits. Computed tomographic (CT) scanning of the spine showed a large heterogeneously attenuating area in the paraspinal tissue at the level of T3-T4 along with a large nodule in the left upper lung. Bone scan revealed abnormalities in the thoracic spine with increased uptake in the right sacroiliac (SI) joint. The differential diagnosis included sarcoidosis, tuberculosis, brucellosis, and disseminated fungal infection. Further investigations were pursued to identify the causal etiology.

The lung nodule was biopsied and smear-negative for acid-fast bacilli, and culture negative for fungus, bacteria, and mycobacteria. Histopathology reported caseating granulomas and fungal elements resembling budding yeast. A CT scan of her head revealed no abnormalities. Bronchoalveolar lavage (BAL) was performed. Mycobacterium tuberculosis complex polymerase chain reaction (PCR) and mycobacterial cultures were negative. Magnetic resonance imaging (MRI) revealed a large soft tissue mass at the spinal levels of T3-T5. Incision and drainage of this large paraspinal abscess was performed, along with a laminectomy at T4 and T5 with rod and pedicle screw placement due to involvement of the adjacent vertebral bodies. Liposomal amphotericin B was started empirically, as the only etiologic clue remained the yeast-like elements identified in the lung nodule biopsy.

Histopathology from the paravertebral collection returned positive for yeast-like cells on PAS and GMS staining, suggestive of histoplasmosis. Bacterial cultures, originally submitted for Brucella, were found to be growing a colony of dark pigmented mold. Microscopy revealed numerous oval shaped conidia with rectangular phialides in keeping with a melanized fungus, identified as Exophiala species (Fig. 1). Fontana Masson staining confirmed the presence of pigmented fungi in original pathology specimens (Fig. 2). Voriconazole was added empirically and liposomal amphotericin B was continued. The patient developed progressive pain in her left shoulder and right SI joint. MRI of the pelvis revealed progression of an osteolytic lesion in the right iliac crest and fluid accumulation in the right sacro-iliac joint. Her therapy was changed from voriconazole to posaconazole empirically at $300 \mathrm{mg}$ IV daily given her ongoing disease progression.

Multiple operative cultures and the BAL sample became positive for dark pigmented mold. Sequencing of the D1-D2 region of the large (28S) ribosomal subunit of the isolate resulted in $98.6 \%$ similarity to Exophiala dermatitidis, confirming a diagnosis of Exophiala dermatitidis [10]. Susceptibility testing results are listed in Table 1. Due to local availability of oral antifungals, posaconazole was changed to oral voriconazole $200 \mathrm{mg}$ PO, and liposomal amphotericin B was discontinued following one month of combination therapy given the patient had experienced significant clinical improvement. Prior to discharge from hospital, a trough voriconazole level was found to be therapeutic at $2.99 \mathrm{mcg} / \mathrm{mL}$ (target range $2-5 \mathrm{mcg} / \mathrm{mL}$ ).

Three weeks following discharge, she again presented with an enlarging mass over her left shoulder blade and ultrasound revealed a thick-walled hyper-dense lesion. A

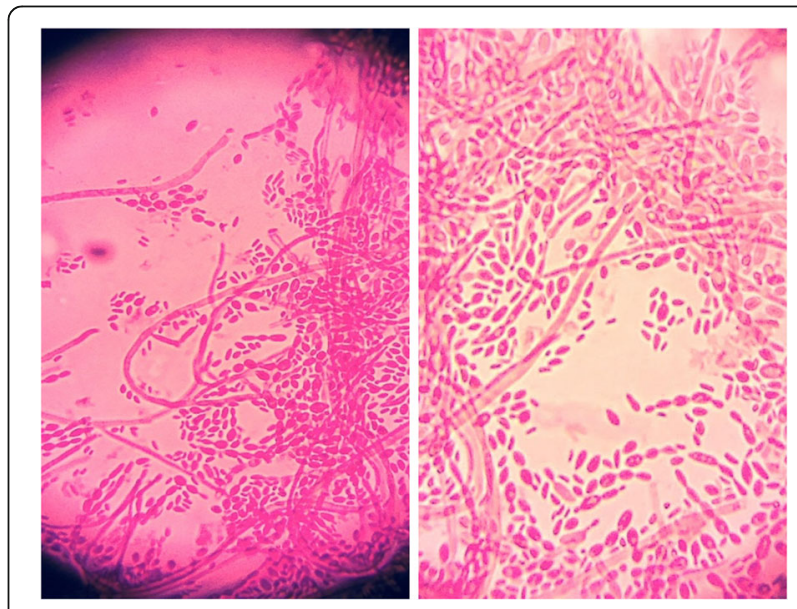

Fig. 1 Exophiala dermatitidis isolated from surgically debrided paravertebral tissue. Lactofuchsin mount, 400X 


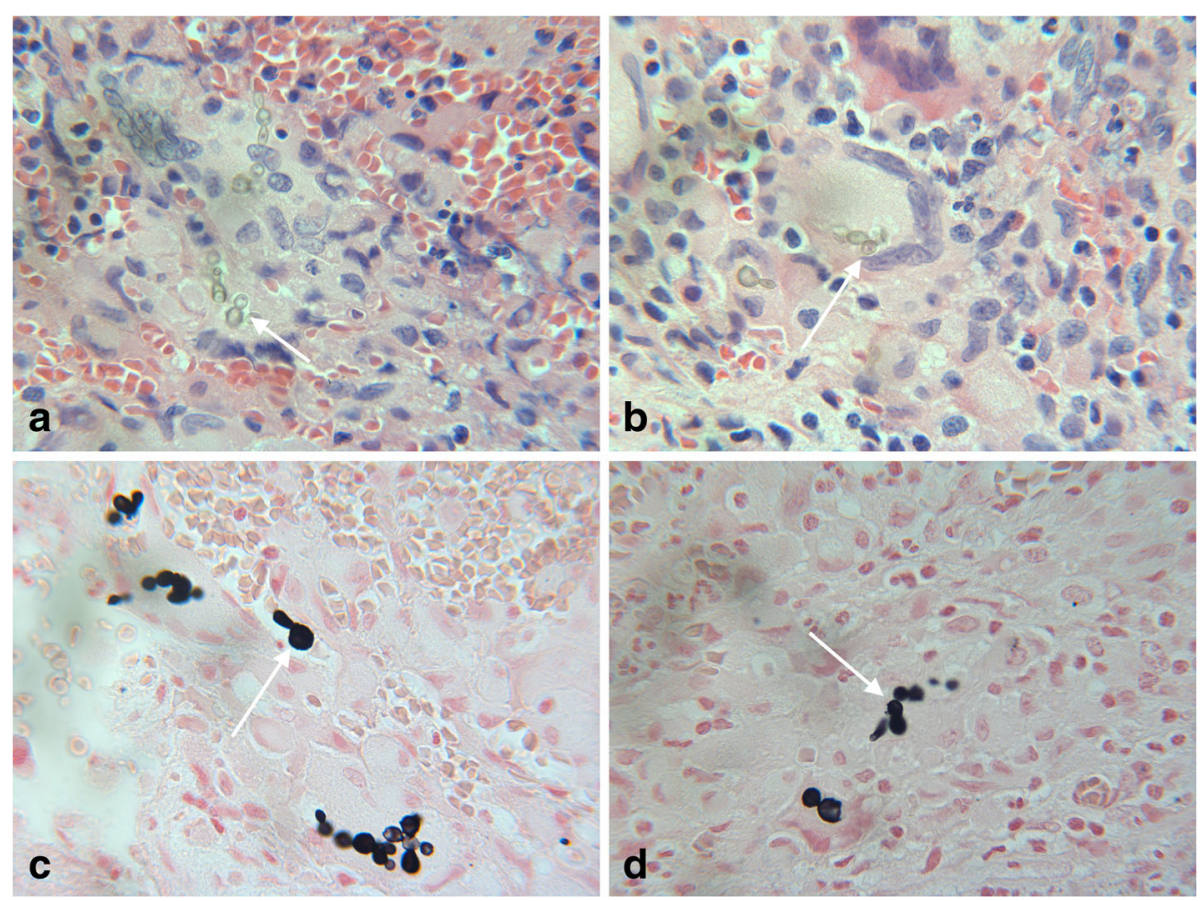

Fig. 2 a, b Fungal elements visualized in surgically debrided paravertebral tissue, identified from culture as Exophiala dermatitidis. H\&E staining, 400X. c, d Fungal elements visualized in surgically debrided paravertebral tissue, identified from culture as Exophiala dermatitidis. Fontana-Masson stain, $400 \mathrm{X}$

spontaneously draining sinus from her thoracolumbar incision was noted. Repeat MRI of her spine and pelvis revealed a bone marrow signal in the right iliac region $(7.4 \times 2.8 \mathrm{~cm})$ and large peripherally enhancing fluid collections extending bilaterally down the spine, enveloping the spinal hardware. She was readmitted for irrigation and debridement of these collections. Voriconazole was discontinued, and combination therapy with liposomal amphotericin B $200 \mathrm{mg}$ IV daily and posaconazole $300 \mathrm{mg}$ IV daily was resumed.

She continued to have daily fevers with worsening pain and effusion of her left shoulder. MRI demonstrated dramatic changes suggestive of a highly aggressive process involving the right iliac crest and left proximal humerus. Her spinal hardware was removed and the surrounding area extensively debrided along with an excisional arthroplasty of the left shoulder. Operative cultures remained negative despite extended incubation. She

Table 1 Antifungal Susceptibility Testing of Exophiala dermatitidis isolate

\begin{tabular}{ll}
\hline Antifungal & MIC $(\mathrm{mg} / \mathrm{L})$ \\
\hline 5-Flucytosine & 0.12 \\
Amphotericin B & 0.5 \\
Itraconazole & 0.25 \\
Posaconazole & 0.12 \\
Voriconazole & 0.12 \\
\hline
\end{tabular}

remained on posaconazole and liposomal amphotericin $\mathrm{B}$ throughout hospitalization and received a six-week course of combination therapy. In total, she required 7 surgical interventions.

Two weeks following discharge, liposomal amphotericin B was discontinued, and posaconazole was continued at $400 \mathrm{mg}$ orally once daily. After receiving nearly 500 days of antifungal therapy following her last surgery, with no clinical evidence of infection and normalized lab parameters, her posaconazole was discontinued. Over 1.5 years following discontinuation of antifungal therapy, she remains disease free with normalized inflammatory markers and continues to be followed closely by the infectious disease team. She has successfully received a shoulder prosthesis to regain significant function in her left arm.

\section{Discussion and conclusions}

Several different species of Exophiala have been documented, with $E$. dermatitidis being known to cause cutaneous and subcutaneous phaeohyphomycosis [7]. Systemic infections are rare with an extensive literature review in 2013 documenting 40 cases [5, 8, 9]. Despite identification of $E$. dermatitidis in environmental samples globally, disseminated human infection is extremely rare in North America and nearly all cases are reported from Asia [7, 9]. Environmental samples have been noted in abundance in public steam baths, and water 
reservoirs, which may relate to the geographic differences in infection [11]. In this case, it is believed that the patient contracted the infection while residing in India. Extra-cutaneous manifestations include lymphadenitis, fungemia, cerebral infections, stomatitis, otitis media, corneal ulcers, esophagitis, pneumonia, liver cirrhosis, pancreatitis, inflammation of the gastrointestinal and biliary systems, endocarditis and peritonitis $[2,4,9,12]$. Li et al. published a case series of seven fatal Exophiala sp. infections in China, two of which had bone involvement, however speciation revealed Exophiala spinifera as the etiologic agent [13]. E. dermatitidis has not previously been documented to cause bone or joint involvement.

Of all reported cases, approximately $70 \%$ have an identifiable underlying risk factor such as an underlying immunocompromised state, intravenous drug use, long-term catheters, malignancy or cystic fibrosis $[1,9]$. Case reports reveal that patients often present with hepatomegaly and lymphadenopathy $[3,5,7]$. Biopsies of liver and lymph nodes frequently show granulomas, which may lead to an early misdiagnosis of sarcoidosis, lymphoma or tuberculosis $[2,3]$.

Most infections initially involve the skin and are suspected to spread hematogenously [7]. The initial skin lesions our patient presented with may have represented cutaneous or subcutaneous fungal infection. Several case reports have described patients being diagnosed with tinea versicolor prior to dissemination with $E$. dermatitidis, leading to the belief that inoculation occurred initially through the skin [3-5]. Since initial biopsies revealed non-specific granulomatous changes, empiric steroids and immunosuppressive agents initiated for sarcoidosis may have facilitated further dissemination of the organism.

E. dermatitidis grows slowly and appears only after prolonged incubation of up to seven days, with one study suggesting cultures be held for at least four weeks $[2,14]$. The organism may be missed if fungal cultures are not performed. Accurate identification of Exophiala species is enabled by molecular diagnostics involving sequencing of the internal transcribed space (ITS) region of the ribosomal DNA [6].

A review of 20 disseminated infections from 1960 to 1992 revealed a mortality rate of $48 \%$, however a subsequent review between 1993 and 2011 based on 24 cases revealed a mortality rate of $25 \%[1,5]$. Improved outcomes are believed to be due to the availability of improved antifungal therapies. If CNS involvement develops, the survival rate is $20 \%$ [1].

No large-scale controlled analyses of treatment options are available. Amphotericin B, flucytosine, itraconazole, voriconazole and posaconazole have all been used with varying degrees of success [2, 15]. In 2011, Badali et al. performed a study comparing 11 different antifungal drug susceptibilities to $E$. dermatitidis and noted that the MICs were similar between environmental and clinical strains of the organism as well as isolates from Asia, America and Europe [16]. The lowest MICs were observed to posaconazole and itraconazole followed by voriconazole $[15,16]$. Fluconazole and amphotericin B appear to have poor activity against $E$. dermatitidis $[2,14,16]$.

Combination therapy of caspofungin with voriconazole, amphotericin or itraconazole may facilitate synergistic activity against $E$. dermatitidis, although monotherapy with an echinocandin is not recommended $[14,16]$. Both voriconazole and posaconazole have good cerebral penetration, ideal for treating disseminated infections. There is limited data regarding the in vivo efficacy of antifungal therapy and there are no defined breakpoints available for antifungal agents [17]. In deep-seated infections, long-term survival has only been reported when complete surgical resection is obtained, however prognosis remains poor $[15,17]$. The decision to discontinue therapy was based on demonstrated clinical stability, normalized lab parameters, expert opinion, and extensive discussion with the patient and her immediate family members.

\section{Key messages}

1) Described is the first identified case of disseminated Exophiala dermatitidis causing osteomyelitis and septic arthritis in a patient on immunosuppressive therapy.

2) Exophiala dermatitidis is a dematiaceous fungus isolated from environmental sources.

3) Systemic infections are very rarely caused by $E$. dermatitidis, however when present are associated with significant morbidity and mortality.

4) Exophiala dermatitidis grows slowly and therefore fungal cultures need to be held for prolonged incubation or the organism may be missed.

5) Through aggressive surgical intervention and prolonged antifungal therapy, a positive outcome may be achieved in disseminated $E$. dermatitidis infections.

Abbreviations

BAL: Bronchoalveolar lavage; CT: Computed tomography; GMS: Grocott's methenamine silver stain; ITS: Internal transcribed space; MIC: Minimum inhibitory concentration; MRI: Magnetic resonance imaging; PAS: Periodic acid-Schiff stain; PCR: Polymerase chain reaction; SAT: Serum agglutination testing; SI: Sacroiliac joint; S-LPS: Smooth lipopolysaccharide

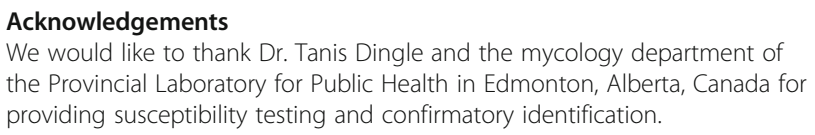

Availability of data and materials

Data sharing is not applicable to this article as no datasets were generated or analyzed during the current study. 


\section{Authors' contributions}

RL was involved in the literature review, planning and writing of the manuscript. JM was the medical microbiologist who performed the microbiological analysis of this case. SS was involved in patient care as well as reviewing the manuscript. AW was the lead physician involved in patient care as well as planning, writing and reviewing the manuscript. All authors read and approved the final manuscript.

\section{Ethics approval and consent to participate}

Not applicable.

\section{Consent for publication}

Written informed consent was obtained from the patient for publication of this case report and any accompanying images. A copy of the written consent is available for review by the Editor of this journal.

\section{Competing interests}

The authors declare that they have no competing interests.

\section{Publisher's Note}

Springer Nature remains neutral with regard to jurisdictional claims in published maps and institutional affiliations.

\section{Author details}

'Department of Medicine, University of Calgary, Calgary, Alberta, Canada. ${ }^{2}$ Regina Qu'Appelle Health Region, Department of Laboratory Medicine, University of Saskatchewan, College of Medicine, Regina, Saskatchewan, Canada. ${ }^{3}$ Division of Infectious Diseases, Department of Medicine, University of Saskatchewan, Regina, Saskatchewan, Canada. ${ }^{4} 4 \mathrm{E}$ - ID Clinic, Regina General Hospital, 1440 14th Avenue Regina, Regina, Saskatchewan, Canada.

Received: 14 March 2018 Accepted: 28 May 2018

Published online: 04 June 2018

\section{References}

1. Suzuki K, Nakamura A, Fujieda A, Nakase K, Katayama N. Pulmonary infection caused by Exophiala dermatitidis in a patient with multiple myeloma: A case report and a review of the literature. Med Mycol Case Rep. 2012;1:95-8.

2. Alabaz D, Kibar F, Arikan S, Sancak B, Celik U, Aksaray N, et al. Systemic phaeohyphomycosis due to Exophiala (Wangiella) in an immunocompetent child. Med Mycol. 2009;47(6):653-7.

3. Oztas E, Odemis B, Kekilli M, Kurt M, Dinc BM, Parlak E, et al. Systemic phaeohyphomycosis resembling primary sclerosing cholangitis caused by Exophiala dermatitidis. J Med Microbiol. 2009;58:1243-6.

4. Hiruma M, Kawada A, Ohata H, Ohnishi Y, Takahashi H, Yamazaki M, et al. Systemic phaeohyphomycosis caused by Exophiala dermatitidis. Mycoses. 1993;36(1):2:1-7.

5. Matsumoto T, Matsuda T, McGinnis MR, Ajello L. Clinical and mycological spectra of Wangiella dermatitidis infections. Mycoses. 1993;36:145-55.

6. Silva WC, Gonçalves SS, Santos DW, Padovan AC, Bizerra FC, Melo AS. Species diversity, antifungal susceptibility and phenotypic and genotypic characterisation of Exophiala spp. infecting patients in different medical centres in Brazil. Mycoses. 2017;60(5):328-37.

7. Kenney RT, Kwon-Chung KJ, Waytes AT, Melnick DA, Pass HI, Merino MJ, et al. Successful treatment of systemic Exophiala dermatitidis infection in a patient with chronic granulomatous disease. Clin Infec Dis. 1992; 14(1):235-42.

8. Chen M, Zhang J, Dong Z, Wang F. Cutaneous phaeohyphomycosis caused by Exophiala dermatitidis: A case report and literature review. Indian J Dermatol Venereol Leprol. 2016;82(2):173-7.

9. Patel AK, Patel KK, Darji P, Sinqh R, Shivaprakash MR, Chakrabarti A. Exophiala dermatitidis endocarditis on native aortic valve in a postrenal transplant patient and review of literature on E.Dermatitidis Infections. Mycoses. 2013;56:365-72.

10. Clinical and Laboratory Standards Institute (CLSI). Interpretive Criteria for Identification of Bacteria and Fungi by DNA Target Sequencing, 1st Edition. CLSI document MM18-A. 2008. https:/clsi.org/standards/products/ molecular-methods/documents/mm18/. Accessed 23 Oct 2017.

11. Matos T, de Hoog GS, de Boer AG, de Crom I, Haase G. High prevalence of the neurotrope Exophiala dermatitidis and related oligotrophic black yeasts in sauna facilities. Mycoses. 2002;45:373-7.
12. Gold WL, Vellend H, Salit IE, Campbell I, Summerbell R, Rinaldi M, et al. Successful treatment of systemic and local infections due to Exophiala species. Clin Infect Dis. 1994;19:339-41.

13. Li DM, Li RY, de Hoog GS, Sudhadham M, Wang DL. Fatal Exophiala infections in China, with a report of seven cases. Mycoses. 2011;54(4):e136-42.

14. Horre R, Schaal KP, Siekmeier R, Sterzik B, de Hoog GS, Schnitzler N. Isolation of fungi, especially Exophiala dermatitidis, in patients suffering from cystic fibrosis. A prospective Study. Respiration. 2004;71(4):360-6.

15. Sun Y, Liu W, Wan Z, Wang X, Li R. Antifungal activity of antifungal drugs, as well as drug combinations against Exophiala dermatitidis. Mycopathologia. 2011:171(2):111-7.

16. Badali H, De Hoog GS, Sudhadham M, Meis JF. Microdilution in vitro antifungal susceptibility of Exophiala dermatitidis a systemic opportunist. Med Mycol. 2011;49(8):819-24.

17. Zheng JS, Sutton DA, Fothergill AW, Rinaldi MG, Harrack MJ, de Hoog GS, Spectrum of clinically relevant Exophiala species in the United States. J Clin Microbiol. 2007:45(11):3713-20.

\section{Ready to submit your research? Choose BMC and benefit from:}

- fast, convenient online submission

- thorough peer review by experienced researchers in your field

- rapid publication on acceptance

- support for research data, including large and complex data types

- gold Open Access which fosters wider collaboration and increased citations

- maximum visibility for your research: over $100 \mathrm{M}$ website views per year

At BMC, research is always in progress.

Learn more biomedcentral.com/submissions 